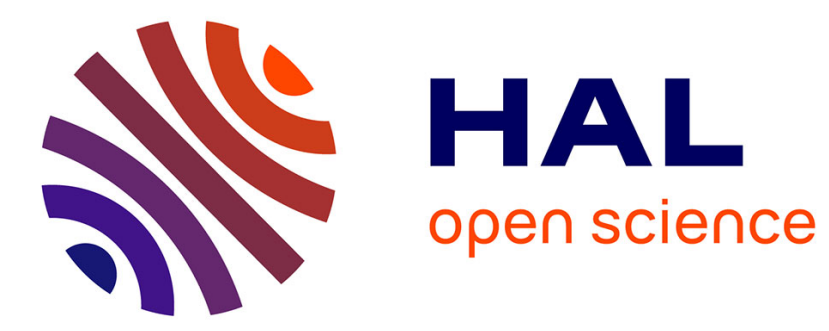

\title{
Capacity drops at merges: new analytical investigations
}

\author{
Ludovic Leclercq, Victor L. Knoop, Florian Marczak, Serge P. Hoogendoorn
}

\section{To cite this version:}

Ludovic Leclercq, Victor L. Knoop, Florian Marczak, Serge P. Hoogendoorn. Capacity drops at merges: new analytical investigations. Transportation research. Part C, Emerging technologies, 2016, 62, pp.171-181. 10.1016/j.trc.2015.06.025 . hal-01215753v2

\section{HAL Id: hal-01215753 \\ https://hal.science/hal-01215753v2}

Submitted on 1 Jun 2017

HAL is a multi-disciplinary open access archive for the deposit and dissemination of scientific research documents, whether they are published or not. The documents may come from teaching and research institutions in France or abroad, or from public or private research centers.
L'archive ouverte pluridisciplinaire HAL, est destinée au dépôt et à la diffusion de documents scientifiques de niveau recherche, publiés ou non, émanant des établissements d'enseignement et de recherche français ou étrangers, des laboratoires publics ou privés. 


\title{
Capacity drops at merges: new analytical investigations
}

\author{
Ludovic Leclercq (Corresponding author) \\ Professor \\ Université de Lyon, Lyon, France \\ IFSTTAR, LICIT, Bron, France \\ ENTPE, LICIT, Vaulx-en-Velin, France \\ ludovic.leclercq@entpe.fr
}

\author{
Victor L. Knoop \\ Assistant Professor \\ Delft University of Technology \\ Stevinweg 1 \\ Delft, The Netherlands \\ v.1.knoop@tudelft.nl

\section{Florian Marczak \\ PhD Student} \\ Université de Lyon, Lyon, France \\ IFSTTAR, LICIT, Bron, France \\ ENTPE, LICIT, Vaulx-en-Velin, France \\ florian.marczak@entpe.fr
}

Serge P. Hoogendoorn

Professor

Delft University of Technology

Stevinweg 1

Delft, The Netherlands

s.p.hoogendoorn@tudelft.nl 


\begin{abstract}
This paper focuses on the derivation of analytical formulae to estimate the effective capacity at freeway merges. It extends previous works by proposing a generic framework able to account for (i) heterogeneous vehicle characteristics and (ii) refined description of the physical interactions between upstream waves and downstream voids created by inserting vehicles within the merge area. The provided analytical formulae permit to directly compute the capacity values when the merge is self-active, i.e. when both upstream roads are congested while downstream traffic conditions are free-flow. They show that accounting for vehicle heterogeneity is not necessary when only the mean capacity is targeted. Calculations with the proper mean value for all parameters provide almost the same results as calculations that consider the full distributions for all parameters. This means that calibrating all distributions is not necessary only the mean parameter values are important. Finally, this paper also shows that vehicle heterogeneity plays a major role in the flow dynamics just upstream of the merge.
\end{abstract}

\title{
Keywords:
}

Active bottleneck, Capacity Drop, Freeway Merge, Kinemactic Wave, Heterogeneous Vehicles. 


\section{Introduction}

Determing the effective merge capacity, i.e. the maximum flow that can be observed just downstream of freeway merges, is crucial for traffic operations. This is not only important for simulation purpose but also to develop better control strategies. Effective capacity is referred in some papers as the queue discharge rate. Experimental findings show that capacity drops are often observed at merges even if downstream traffic conditions are in free-flow, e.g. (Cassidy and Bertini, 1999; Kerner, 2002; Chung et al., 2007; Sarvi et al., 2007; Zheng et al., 2011). The magnitude of the capacity drops is mentioned to be between 10 to $30 \%$ of the maximal observed flow. The main physical explanations for such a phenomenon are lower speeds for merging vehicles combined with bounded acceleration, e.g. (Cassidy and Rudjanakanoknad, 2005; Laval et al., 2005; Treiber et al., 2006; Laval and Daganzo, 2006), and the impacts of driver behaviors, e.g. (Cassidy and Ahn, 2005; Coifman and Kim, 2011; Chen et al., 2014). In a nutshell, slower vehicles create voids in front of them that locally reduce the available capacity and lead to temporal flow restrictions. It is important to notice that driver relaxation few hundred meters downstream of the merge point and the related global acceleration process may also trigger capacity drops (Kim and Coifman, 2013; Carlson et al, 2014). In this paper, we will only focus on the physical process close to the merge, i.e. the impacts of merging vehicles combined with bounded acceleration.

Except for direct experimental observations, the most common way to determine the effective merge capacity is to use a traffic model able to reproduce the underlying physical mechanisms, e.g. (Laval and Daganzo, 2006; Srivastava and Geroliminis, 2013). This requires running a simulation for every new set of parameters and is not really convenient when looking for a first and quick approximation of how a merge behaves or to determine which parameters are the most influential, e.g. for sensitivity analysis. (Laval, 2006) is one of the first attempt to estimate capacity related to a dynamic and local physical process. To the authors' knowledge, (Leclercq et al., 2011) is the only attempt to derive an analytical expression that explicitly relates the merge effective capacity to the different parameters. This expression is derived by considering that inserting vehicles act as moving bottlenecks (Newell, 1998; Leclercq et al., 2004) with bounded acceleration while mainstream vehicles behave according to the kinematic wave model (Lighthill and Whitham, 1955; Richards, 1956) with a triangular fundamental diagram. The central point of this contribution is to handle the interactions between moving bottlenecks when vehicles insert at different location along the on-ramp.

This first attempt has two main shortcomings. First, vehicle characteristics are supposed homogeneous, i.e. same acceleration, same jam spacing... Second, interactions of upstream propagating traffic waves with downstream propagating voids created downstream of moving bottlenecks are neglected. This paper proposes new analytical investigations that tackle these two shortcomings. Notably heterogeneous vehicle characteristics will be introduced to account for traffic composition (trucks and cars) but also for driver behaviors (random maximal acceleration). As a major result an updated expression for the effective capacity defined by eq. (5) in (Leclercq et al., 2011) will be established. In this paper, we will assume that both the on-ramp and the freeway are congested upstream of the merge. (Leclercq et al., 2011) provides all the materials to extend the results to situations when the on-ramp is in free-flow. Furthermore, we will consider that the inserting flow $q_{0}$ is given when calculating the merge effective capacity $C$. One more time, the major challenge is to derive an update version of eq. (5) in (Leclercq et al., 2011). Then, all methodology already presented in (Leclercq et al., 2011) can be directly applied. Notably, when the merge ratio $\alpha$ is given (Daganzo, 1995), $q_{0}$ can be derived by solving eq. (1). This provides both equilibrium traffic states upstream of a self-active merge, i.e. when the congestion is not coming from downstream. Finally, note that we will restrict our investigations here to a one-lane freeway. Extensions to multi-lane freeways have already been discussed in (Leclercq et al., 2011). The corresponding methods are directly applicable to the extended expression of the effective capacity.

$$
(1+1 / \alpha) q_{0}=C\left(q_{0}\right)
$$

This paper is organized as follow: the first section proposes a generic expression for the effective capacity. Section 2 deals with proper consideration of voids downstream of moving bottlenecks while 


\section{Leclercq et al}

section 3 addresses the question of heterogeneous vehicle characteristics. The main work in these two sections is to derive the characteristics of the statistical distributions that appear in the generic expression. The main challenge is to maintain analytical tractability from end-to-end. Analytical expressions will be compared to numerical simulations to test the relevance of the required approximations. The last section presents a brief discussion.

\section{Generic expression for the effective capacity}

Consider a merge with two one-lane roads. Vehicle $i$ inserts from the on-ramp at time $t_{i}$ and location $x_{i}$ $\left(0 \leq x_{i} \leq L\right)$, where $L$ is the length of the insertion lane, see Figure 1a. The time headway $h_{i}=t_{i+1}-t_{i}$ between two successive insertions follows an unknown distribution $H\left(h_{0}, s_{H}\right)$ with mean $h_{0}=1 / q_{0}$ and standard deviation $s_{H}$. Inserting vehicles are considered as moving bottlenecks (Newell, 1998; Leclercq et al., 2004) on the freeway with initial speed $v_{0, i}$ and bounded acceleration $a_{i}$. The distributions of these parameters are respectively described by $V_{0}\left(v_{0}, s_{V 0}\right)$ and $A\left(a, s_{A}\right)$. Note that capital letters will be used for defining the distributions associated to random variables labeled with lower case letters. Platoons of vehicles upstream of each moving bottleneck on the main road are described by the kinematic wave model (Lighthill and Whitham, 1955; Richards, 1956) and a triangular fundamental diagram with wave speed $w$ and jam density $\kappa_{i}$. Free-flow speed has no influence here and it seems reasonable for freeway traffic to assume same wave speeds for all platoons (Chiabaut et al., 2010). A different jam density value is assigned to each inserting vehicles following $K\left(\kappa, s_{K}\right)$. In this paper, we will assume that this value also characterizes the mean jam density of the platoons leaded by the inserting vehicle.

(b)
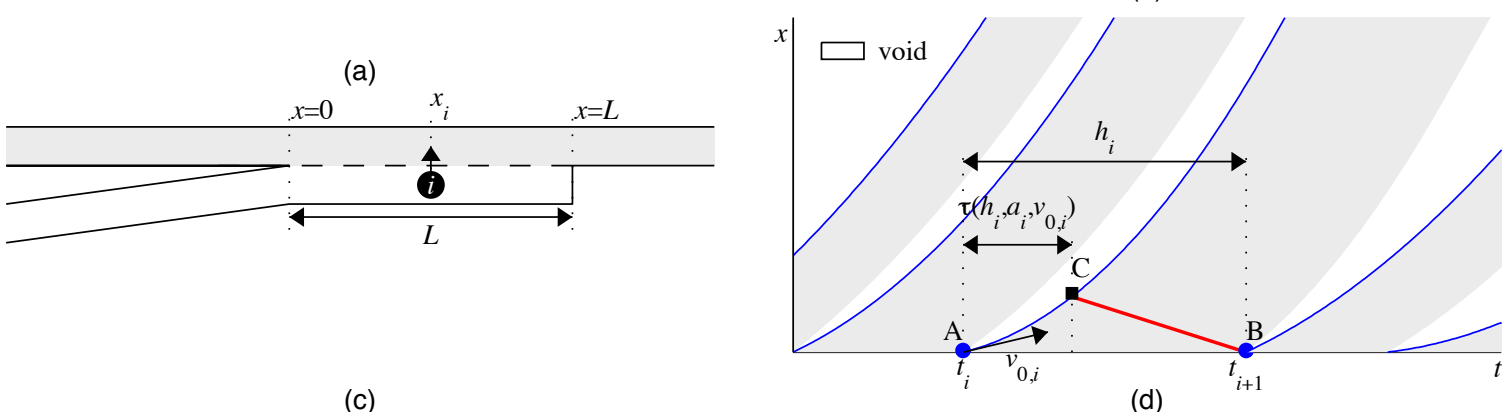

(c)
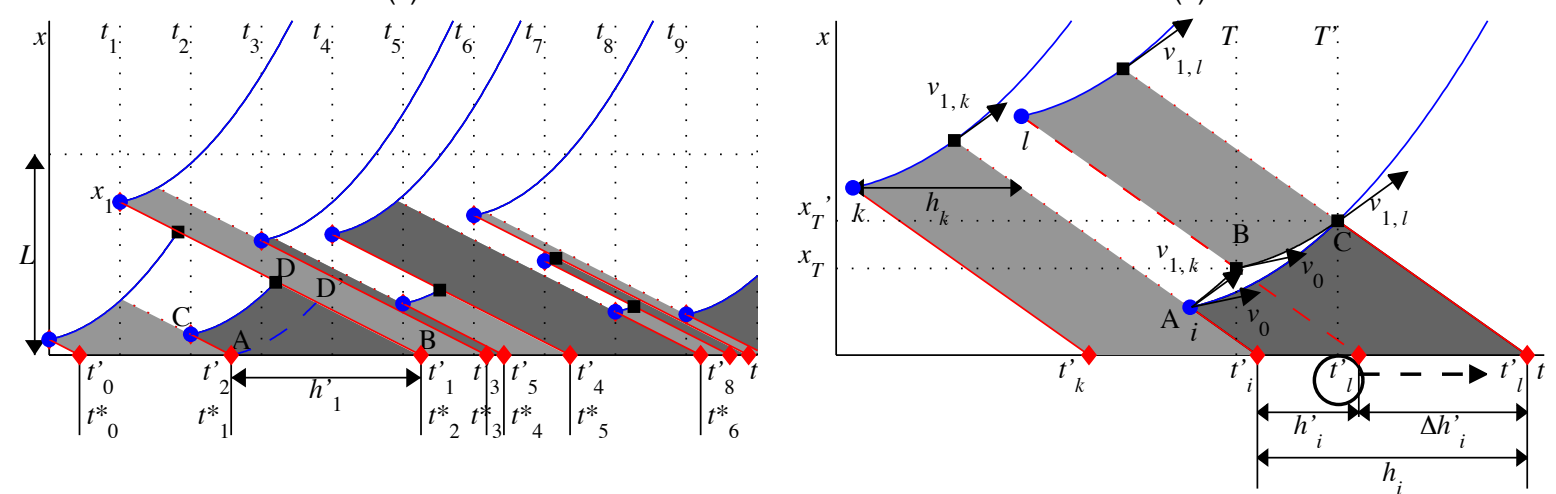

Figure 1: (a) Sketch of the merge (b) Inserting process when $L=0$ (c) Inserting process when $L>0$, no interaction between waves and voids (d) Accounting for interactions between waves and voids.

To establish the generic expression for the effective capacity $C$, vehicles are first assumed to all insert at $x=0$, i.e. $L=0$, see Figure $1 \mathrm{~b}$. Let $\delta_{i}$ be the cumulative number of vehicles that have crossed $x=0$ between time $t_{i}$ and $t_{i+1}$. Variational theory (Daganzo, 2005) states that $\delta_{i}$ can be equally calculated on the paths $\mathrm{A} \rightarrow \mathrm{B}$ or $\mathrm{A} \rightarrow \mathrm{C} \rightarrow \mathrm{B}$, see Fig. 1b. This can also be seen as a direct application of the Green's theorem. No vehicle can pass the bottleneck between $\mathrm{A}$ and $\mathrm{C}$, so $\delta_{i}$ is equal to $w \kappa_{i}\left(h_{i}-\tau_{i}\right)$, where $\tau_{i}$ is the time duration between points $\mathrm{A}$ and $\mathrm{C}$. The effective capacity $C$ corresponds to the ratio between the sum of $\delta_{i}$ and the total duration of the process, i.e. the sum of $h_{i}$, when the number of insertions tends to infinity. It is then given by: 


$$
\begin{gathered}
C=\sum_{i=1}^{n \rightarrow \infty} \delta_{i} / \sum_{i=1}^{n \rightarrow \infty} h_{i}=w \sum_{i=1}^{n \rightarrow \infty} \kappa_{i}\left(h_{i}-\tau\left(h_{i}, v_{0, i}, a_{i}\right)\right) / \sum_{i=1}^{n \rightarrow \infty} h_{i} \\
\tau=\frac{1}{a_{i}}\left(-w-v_{0, i}+v\right) ; v\left(h_{i}, v_{0, i}, a_{i}\right)=\sqrt{\left(w+v_{0, i}\right)^{2}+2 w a_{i} h_{i}}
\end{gathered}
$$

Note that the unit of $v$ is consistent with a speed but this expression is not related to any physical definition. The law of large numbers tells us that $(1 / n) \Sigma \delta_{i}$ and $(1 / n) \Sigma h_{i}$ respectively converge to $\Delta$ and $h_{0}$, i.e. the mean of the corresponding distributions. $\Delta$ can be approximated using the multivariate generalization of the Delta method (Oehlert, 1992). This method consists in performing a second-order Taylor expansion of $\delta_{i}$ around the mean values $h_{0}, v_{0}, a$ and $\kappa$ before applying the sum operator. First order terms disappear because the sum of each parameter divided by $n$ converges to the mean. Only second order terms remain and are weighted by either the standard deviation $s_{X}$ of each distribution $X$ or the covariance $\theta_{X, Y}$ between all $X$ and $Y$. Thus, $\Delta$ is given by:

$$
\frac{\Delta}{w}=\delta\left(h_{0}, v_{0}, a, \kappa\right)+\frac{1}{2} \sum_{X \in\left\{H, V_{0}, A, K\right\}} s_{X}^{2} \frac{\partial^{2} \delta_{i}}{\partial X^{2}}+\sum_{(X, Y) \in\left\{H, V_{0}, A, K\right\}, X \neq Y} \theta_{x, y} \frac{\partial^{2} \delta_{i}}{\partial X \partial Y}
$$

We first assume that $H$ and $V_{0}$ are respectively independent from $A$ and $K$ because they depend on the on-ramp traffic conditions and not on the vehicle characteristics. The covariance between these distributions is then zero. It can easily be verified that the second derivative of $\delta_{i}$ with respect to $K$ is zero. Interestingly, this means that the $K$-distribution has no influence on $C$, only the correlation between $K$ and $A$ does. Finally, all the derivatives of $\delta_{i}$ can be expressed using the derivatives of $\tau$. We then obtain the following generic expression for $C$ :

$$
\begin{aligned}
& C=\frac{w \kappa}{h_{0}}\left(h_{0}-\right. \tau\left(h_{0}, v_{0}, a\right)-\frac{1}{2} s_{H}^{2} \frac{\partial^{2} \tau}{\partial H^{2}}-\frac{1}{2} s_{V_{0}}^{2} \frac{\partial^{2} \tau}{\partial V_{0}{ }^{2}}-\frac{1}{2} s_{A}^{2} \frac{\partial^{2} \tau}{\partial A^{2}}-\theta_{H, V_{0}} \frac{\partial^{2} \tau}{\partial H \partial V_{0}} \\
&\left.-\frac{\theta_{A, \mathrm{~K}}}{\kappa} \frac{\partial \tau}{\partial A}\right)
\end{aligned}
$$

The derivatives of $\tau$ are provided in eq. (5). We will show in section 3 and 4 that introducing more relevant physical hypothesis like $L>0$, interactions between voids and waves or random vehicle characteristics is "just" a question of properly calculating the moments of all distributions.

$$
\begin{gathered}
\frac{\partial^{2} \tau}{\partial H^{2}}=\frac{-a w^{2}}{v^{3}\left(h_{0}, v_{0}, a\right)} ; \frac{\partial^{2} \tau}{\partial V_{0}{ }^{2}}=\frac{2 w h_{0}}{v^{3}\left(h_{0}, v_{0}, a\right)} ; \frac{\partial^{2} \tau}{\partial H \partial V_{0}}=\frac{-w\left(w+v_{0}\right)}{v^{3}\left(h_{0}, v_{0}, a\right)} \\
\frac{\partial \tau}{\partial A}=-\frac{\tau\left(h_{0}, v_{0}, a\right)}{a}+\frac{w h_{0}}{v\left(h_{0}, v_{0}, a\right)} \\
\frac{\partial^{2} \tau}{\partial A^{2}}=\frac{2}{a^{2}}\left(\tau\left(h_{0}, v_{0}, a\right)-\frac{w h_{0}}{v\left(h_{0}, v_{0}, a\right)}-\frac{a w^{2} h_{0}^{2}}{2 v^{3}\left(h_{0}, v_{0}, a\right)}\right)
\end{gathered}
$$

\section{Considering interactions between waves and voids}

In this section, we now consider that insertions can happen anywhere between 0 and $L(L>0)$. Vehicle characteristics are still homogeneous $\left(s_{A}=0\right.$ and $\left.s_{K}=0\right)$. We first show that the physical process with random inserting position can still be described with the generic expression. Second, we look for the analytical expression for the relevant moments in eq. (4). Finally, we derive the full analytical expression.

\subsection{Applying the generic expression}

The general principle for considering insertions at different locations between $x=0$ and $x=L$ has already been presented in (Leclercq et al., 2011). When vehicle $i$ is inserting at time $t_{i}$ and location $x_{i}$, it generates a wave whose speed is $w$ and which carries the speed $v_{0, i}$. This wave reaches $x=0$ at time $t^{\prime}{ }_{i}$, 
see Figure 1c. (Leclercq et al., 2011) explains in details what clearly appears in Figure 1c: the process for determining the effective capacity at $x=0$ is the same when considering $L>0$ or $L=0$. Indeed, the cumulative number of vehicles can be calculated on either paths $\mathrm{A} \rightarrow \mathrm{C} \rightarrow \mathrm{D} \rightarrow \mathrm{B}$ or $\mathrm{A} \rightarrow \mathrm{D}^{\prime} \rightarrow \mathrm{B}$. Calculation on path $\mathrm{A} \rightarrow \mathrm{D}^{\prime} \rightarrow \mathrm{B}$ is exactly the same for the path $\mathrm{A} \rightarrow \mathrm{C} \rightarrow \mathrm{B}$ when $L=0$, see Figure $1 \mathrm{~b} \& \mathrm{c}$. To determine $C$, we only have to replace the distribution $H$ by the distribution $H$ ' where $h_{i}{ }_{i}=t^{*}{ }_{i+1}-t^{*}{ }_{i}$ and $t^{*}$ is the ordered series gained from the realizations of $t^{\prime}$, see Figure $1 \mathrm{c}$. This result resorts to a restrictive assumption: waves coming for moving bottlenecks are not influenced by voids created upstream by other bottlenecks, e.g. wave coming from vehicle 1 propagates until $x=0$ without considering the void created downstream of vehicle 2, see Figure 1c. (Leclercq et al., 2011) mentioned that this assumption helps to keep the analytical calculation simple but they do not investigate how it influences the effective capacity values. This will now be done. Note that (Leclercq et al., 2011) provides the analytical expression for $s_{H}$, when the time between two insertions is set to $h_{0}$, i.e. $s_{H}=0$, and when the distribution of inserting positions in congestion is uniform as suggested by experimental evidence (Daamen et al., 2010):

$$
s_{H^{\prime}}= \begin{cases}L / \sqrt{6} w & \text { if } L \leq w h_{0} \\ h_{0}\left(L-w h_{0} / \sqrt{6}\right) /\left(L+(\sqrt{6}-2) w h_{0}\right) & \text { if } L>w h_{0}\end{cases}
$$

Figure $1 \mathrm{~d}$ shows what happens when considering the interactions between voids and waves. The wave coming from vehicle $l$ meets the void created downstream of vehicle $i$. The void progressively vanishes and the wave can only propagate further downstream when the void has disappeared, i.e. at time $T$ ' in Figure 1d. This changes the time $t^{\prime}{ }_{l}$ when the wave reaches $x=0$ and potentially influences the $H^{\prime}$ distribution that is now simply relabeled $H$. This also modifies the $V_{0}$ distribution. In fact, the initial speed when calculating the cumulative vehicle number between $t^{\prime}{ }_{l}$ and the time when the next wave arrives at $x=0$ is no longer equal to $v_{0, l}$ but is now equal to $v_{1, l}$, see Figure $1 \mathrm{~d}$. $v_{1, l}$ corresponds to the speed carried by the wave coming from vehicle $l$ and that goes through the point $\mathrm{C}$ where the void created by $i$ disappears. The position of this point depends on the initial speed $v_{1, k}$ of the void downstream boundary. This last speed can be determined by identifying the vehicle $k$ that determines the speed profile when $i$ is inserting, see Figure $1 \mathrm{~d}$.

All the challenge is to maintain analytical tractability when calculating the new moments of the $H$ and $V_{0}$ distributions considering the extended physical process with voids. In order to validate the analytical simplifications that we will made, we have developed a numerical code that, for a given $h_{0}=1 / q_{0}$, (i) randomly draws the inserting positions for a set of 5000 vehicles, (ii) matches each vehicle $l$ with the corresponding vehicles $i$ and $k$, (iii) makes the proper calculation for the vehicle $i$ void boundaries, (iv) determines the modified values for $t_{l}{ }_{l}$ and $v_{0, l}$. Note that the wave coming from $l$ not necessarily meets a void and is then unaffected. The results of this process will be further referred as numerical simulation. It is important to notice that this does not correspond to classical results from a traffic simulator because here traffic dynamics is not fully reproduced. These simulations correspond to a direct Monte Carlo implementation of the physical processes described in Figure 1c and Figure 1d. They provide samples for $H$ and $V_{0}$ distributions and also allow us to directly estimate $C$. We perform extensive simulation runs but for illustration purposes, most figures of this article are drawn with the following parameters: $w=19.4 \mathrm{~km} / \mathrm{h}, \kappa=130 \mathrm{veh} / \mathrm{km}, a=1.8 \mathrm{~m} / \mathrm{s}^{2}, q_{0}=0.174 \mathrm{veh} / \mathrm{s}$.

\subsection{Determining the moments of the different distributions}

We further assume that all vehicles have the same speed $v_{0}$ when inserting. This speed is associated to $q_{0}$ through the fundamental diagram. This assumption is reasonable because the on-ramp is congested. Section 5 will explain how this can be relaxed. Note that mathematical expectation (mean) of a distribution $X$ is further labeled $E(X)$.

$\boldsymbol{H}$-distribution. Considering interactions between voids and waves does not change the number of waves created. Thus $E(H)$ remains unchanged and is equal to $h_{0}$. The ordering processing when switching from $t^{\prime}$ to $t^{*}$ makes inaccessible the analytical derivation of the $H$-distribution from the distributions of the inserting position and time. In (Leclercq et al., 2011), the analytical expression of $s_{H}$, see eq. (6), has been obtained by considering extreme case when $L$ is very small and very high and by fitting the global expression using extensive numerical simulations. Notably, it appeared that $H^{\prime}$ follow an exponential distribution when $L$ tends to infinity. Here, we apply the same approach. Figure 


\section{Leclercq et al}

2 shows an example for the evolution of $s_{H}$ with respect to $L$ when interactions between voids and waves are considered or not. This figure highlights that the standard deviation of $H$ is unaffected by the modification of $t^{\prime}{ }_{l}$ when waves encounter interactions with upstream voids. The analytical expression eq. (6) remains fully accurate. This has been confirmed by multiple simulation runs using a wide range of parameter values.

Probability for interacting $\boldsymbol{p}_{\text {int }}$. Not all waves meet voids before reaching $x=0$. Before going further in the calculation of the moments, we need to establish an analytical formulation for the probability $p_{\text {int }}$ that a wave starting from a moving bottleneck experiments interactions. Consider a vehicle $i$ that is inserting at time $t_{i}$ and location $x_{i}$. The wave starting from $i$ can interact with a void created by a vehicle that inserted in the close past or that will insert in the near future, see diamond dots in Figure 2c. To maintain the analytical tractability, we will only consider the closest neighbors, i.e. vehicle $i-1$ and $i+1$, see the shaded area in Figure 2c. There will be no interactions if the starting point of vehicle $i$ 1 makes it arrive above $x_{i}$ at time $t_{i}$ and if vehicle $i+1$ is created above the wave coming from $i$ at time $t_{i+1}$. These two conditions should jointly be true and correspond to initial inserting positions for vehicle $i-1$ and $i+1$ along the two green lines in Figure 2c. This means that $x_{i-1}>x_{\mathrm{i}}-0.5 a h_{0}^{2}-v_{0} h_{0}$ and $x_{i+1}>x_{i}-w h_{0}$. Let denote $b_{1}$ and $b_{2}$ respectively the minimum and the maximum between $x_{\mathrm{i}}-0.5 a h_{0}{ }^{2}-v_{0} h_{0}$ and $x_{i}-w h_{0}$. As the inserting position for all vehicles obeys to a uniform distribution, it comes that the conditional probability $P\left(\right.$ no $\left.\mid x_{i}\right)$ of no interaction given $x_{i}$ is:

$$
P\left(\mathrm{no}_{i}\right)= \begin{cases}1 & \text { if } x_{i} \leq b_{1} \\ \left(L-x_{i}-b_{1}\right) / L & \text { if } b_{1}<x_{i} \leq b_{2} \\ \left(L-x_{i}-b_{1}\right)\left(L-x_{i}-b_{2}\right) / L^{2} & \text { if } x_{i}>b_{2}\end{cases}
$$

The law of total probability makes it possible to determine $p_{\text {int }}$ with respect to $P\left(\right.$ no $\left.\mid x_{i}\right)$ noticing that the probability for vehicle $i$ to insert at position $x_{i}$ is $1 / L$ :

$$
p_{\text {int }}=1-\frac{1}{L} \int_{0}^{L} P\left(\text { no } \mid x_{i}\right) d x_{i}
$$

The black curve in Figure $2 \mathrm{~b}$ compares the numerical and analytical results for $p_{\text {int }}$ and different $L$ values in our example case. It appears that the analytical expression is close to the numerical results even if we only consider the two closest neighbors. $p_{\text {int }}$ is underestimated because our approximation neglects interactions with farer neighbors. This result has also been confirmed by extensive simulation runs. 

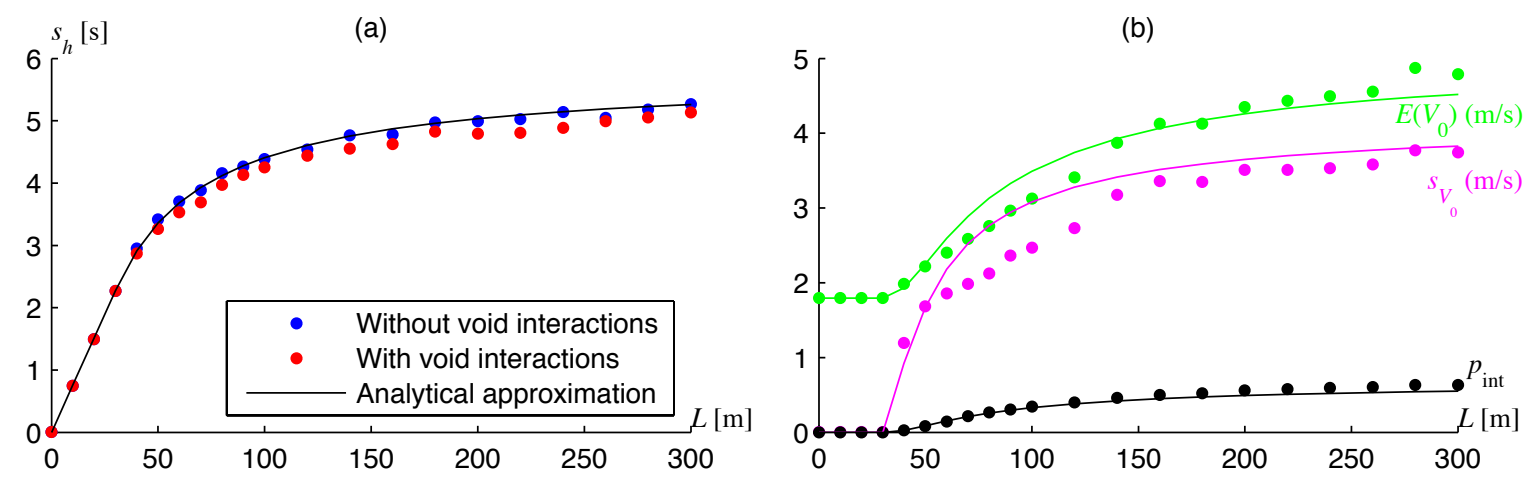

(c)

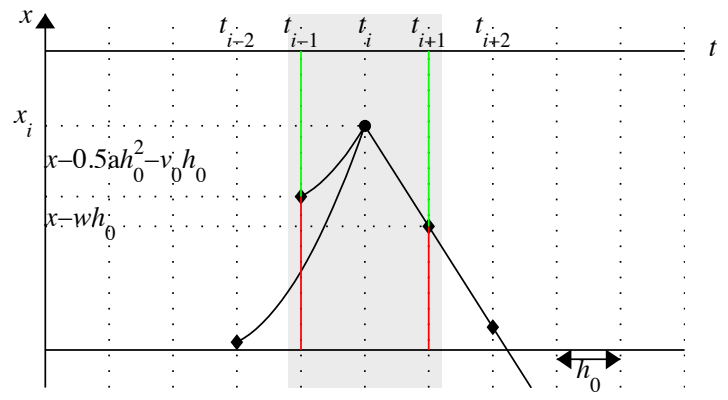

Figure 2: Analytical approximations for (a) the standard deviation of $H$ with and without considering void interactions (b) the mean and the standard deviation of $V_{0}$ and the probability $p_{\text {int }}$ for waves to interact with voids (c) Process for approximating the probability $p_{\text {int }}$.

$\boldsymbol{V}_{0}$-distribution. The initial speed when wave $i$ arrives at $x=0$ may either be equal to $v_{1, l}$ or $v_{0}$ depending on whether an interaction happens or not. We first only consider cases with an interaction. Let denote $\mathrm{A}\left(t_{i}, x_{i}\right)$ the point where the void is created, $\mathrm{B}\left(T, x_{T}\right)$ the point where the wave meets the downstream void boundary and $\mathrm{C}\left(T^{\prime}, x_{T}\right)$ the point where the void disappears, see Figure $1 \mathrm{~d}$. $\mathrm{C}$ is the intersection point of two parabolas corresponding to bounded acceleration trajectories (curves $\mathrm{BC}$ and $\mathrm{AC})$. Solving the associated equations leads to $T^{\prime}=T+\left(v_{1, k}-v_{0}\right) / a$. Equation of curve $\mathrm{BC}$ also tells us that $v_{1, l}=a\left(T^{\prime}-T\right)+v_{0}$. It comes then a very simple result when $v_{0}$ is constant: $v_{1, l}=v_{1, k}$. Finally, $v_{1, l}$ is given by:

$$
v_{1, l}=a \tau\left(h_{k}, v_{0}, a\right)+v_{0}
$$

Eq. (9) means that the $V_{1}$-distribution of only depends on the distribution of $\tau(H)$. Its two first moments are then given by:

$$
\left\{\begin{array}{l}
E\left(V_{1}\right)=a E(\tau(H))+v_{0} \\
E\left(V_{1}^{2}\right)=v_{0}^{2}+2 a E(\tau(H))+a^{2} E\left(\tau^{2}(H)\right)
\end{array}\right.
$$

The mean and standard deviation of the $V_{0}$-distribution can then be derived by applying the law of total expectation with condition probability depending on whether an interaction appears or not:

$$
\begin{gathered}
\left\{\begin{array}{l}
E\left(V_{0}\right)=\left(1-p_{\text {int }}\right) v_{0}+p_{\text {int }} E\left(V_{1}\right) \\
s_{V_{0}}^{2}=E\left(V_{0}^{2}\right)-E\left(V_{0}\right)^{2} ; E\left(V_{0}^{2}\right)=\left(1-p_{\text {int }}\right) v_{0}^{2}+p_{\text {int }} E\left(V_{1}^{2}\right)
\end{array}\right. \\
\Rightarrow\left\{\begin{array}{c}
E\left(V_{0}\right)=v_{0}+a p_{\text {int }} E(\tau(H)) \\
s_{V_{0}}^{2}=a p_{\text {int }}\left(2 E(\tau(H))+a E\left(\tau^{2}(H)\right)\right)
\end{array}\right.
\end{gathered}
$$

The last thing we need to finalize is the calculation of the mathematical expectation of $\tau$ and $\tau^{2}$. This can be achieved by again applying the Delta method (Oehlert, 1992): 


$$
\left\{\begin{array}{l}
E(\tau)=\tau\left(h_{0}, v_{0}, a\right)+\frac{1}{2} s_{H}^{2} \frac{\partial^{2} \tau}{\partial H^{2}} ; \frac{\partial^{2} \tau}{\partial H^{2}} \rightarrow(5) \\
E\left(\tau^{2}\right)=\tau^{2}\left(h_{0}, v_{0}, a\right)+\frac{1}{2} s_{H}^{2} \frac{\partial^{2} \tau^{2}}{\partial H^{2}} ; \frac{\partial^{2} \tau^{2}}{\partial H^{2}}=\frac{2 w^{2}\left(w+v_{0}\right)}{v^{3}\left(h_{0}, v_{0}, a\right)}
\end{array}\right.
$$

Figure $2 \mathrm{~b}$ shows the comparison between the analytical and the numerical calculations for $E\left(V_{0}\right)$ and $s_{V 0}$ and the example case. The results are quite good except for some discrepancies for low $L$ values (between 60 and $150 \mathrm{~m}$ ). The reason is that $v_{1, l}=v_{1, k}$ and eq. (9) hold only if vehicle $l$ inserts outside the void created by vehicle $i$. Otherwise, the void disappears more quickly and $v_{1, l}<v_{1, k}$. Such situations are properly handled in the numerical code but can hardly be introduced in the analytical derivation. Of course, they happen more frequently if the insertion length is small. This explains why the analytical formulas overestimate $E\left(V_{0}\right)$ and $s_{V 0}$ when $L$ is quite small. As usual, extensive simulation runs have been performed to verify that the errors remain in the same level of magnitude. Furthermore, we will see later that such discrepancies have few impacts when calculating $C$.

Covariance between $\boldsymbol{H}$ and $\boldsymbol{V}_{\mathbf{0}}$. To apply eq. (4) with homogeneous vehicle characteristics, the last missing term is $\theta_{H, V 0}$. The analytical derivation of this term is almost impossible because multiple interactions occur. Indeed, when a wave is delayed due to a void this changes the time headways of both neighboring waves and the initial speed for one of them, see Figure 1d. This speed depends on the time headway of another wave associated to vehicle $k$. Because of the ordering process from $t^{\prime}$ to $t^{*}$, it is very difficult to analytically determine the headway index associated to an initial speed modification. Fortunately, when performing the extensive numerical tests it appears that the value of $\theta_{H, V 0}$ remains very low compared to the variances of other distributions whatever the parameters, $q_{0}$ and $L$ are. $H$ and $V_{0}$ are clearly not independent but their covariance can be neglected. $\theta_{H, V 0}$ is then assumed equal to 0 for further analytical calculations.

\subsection{Calculating the effective capacity for different inserting flows}

Figure 3 presents the analytical and numerical results for the effective capacity $C$. Three values for the inserting flow are tested. The blue curve and dots correspond to the case when interactions are neglected and so to the results already stated in (Leclercq et al., 2011). The red curve and dots clearly show the importance of considering the interactions between voids and waves. The estimation of the effective capacity increases up to $15 \%$ when this phenomenon is taken into account. This is explained by the fact that voids created by upstream inserting vehicles reduce the impacts of other vehicles that insert downstream. This tends to increase the capacity.

The effect of interactions starts being noticeable when $L>50 \mathrm{~m}$ except for the lowest $q_{0}$ value. This is because when all vehicles insert on a short distance, waves quickly reaches $x=0$ and do not interact with voids. Another interesting result is that the effective capacity stops significantly increasing when $L$ becomes higher than $150 \mathrm{~m}$. However, the influence of $L$ is important for the lowest values. For example, the effective capacity increases about 15 to $20 \%$ when L increases from 20 to $160 \mathrm{~m}$. This may be interesting for road design.

The most important insight in Figure 3 is that the extended analytical formula performed well whatever the $q_{0}$ and $L$ values are. The discrepancies with the numerical results are always below $3 \%$. This means that eq. (4) provides a very good estimate for the effective capacity even if we resort to restrictive assumptions when determining the moment of some distributions. This is really appealing because this formula provides a direct estimate for the effective capacity without requiring any complex simulation runs. It will now be further extended to account for heterogeneous vehicle characteristics. 


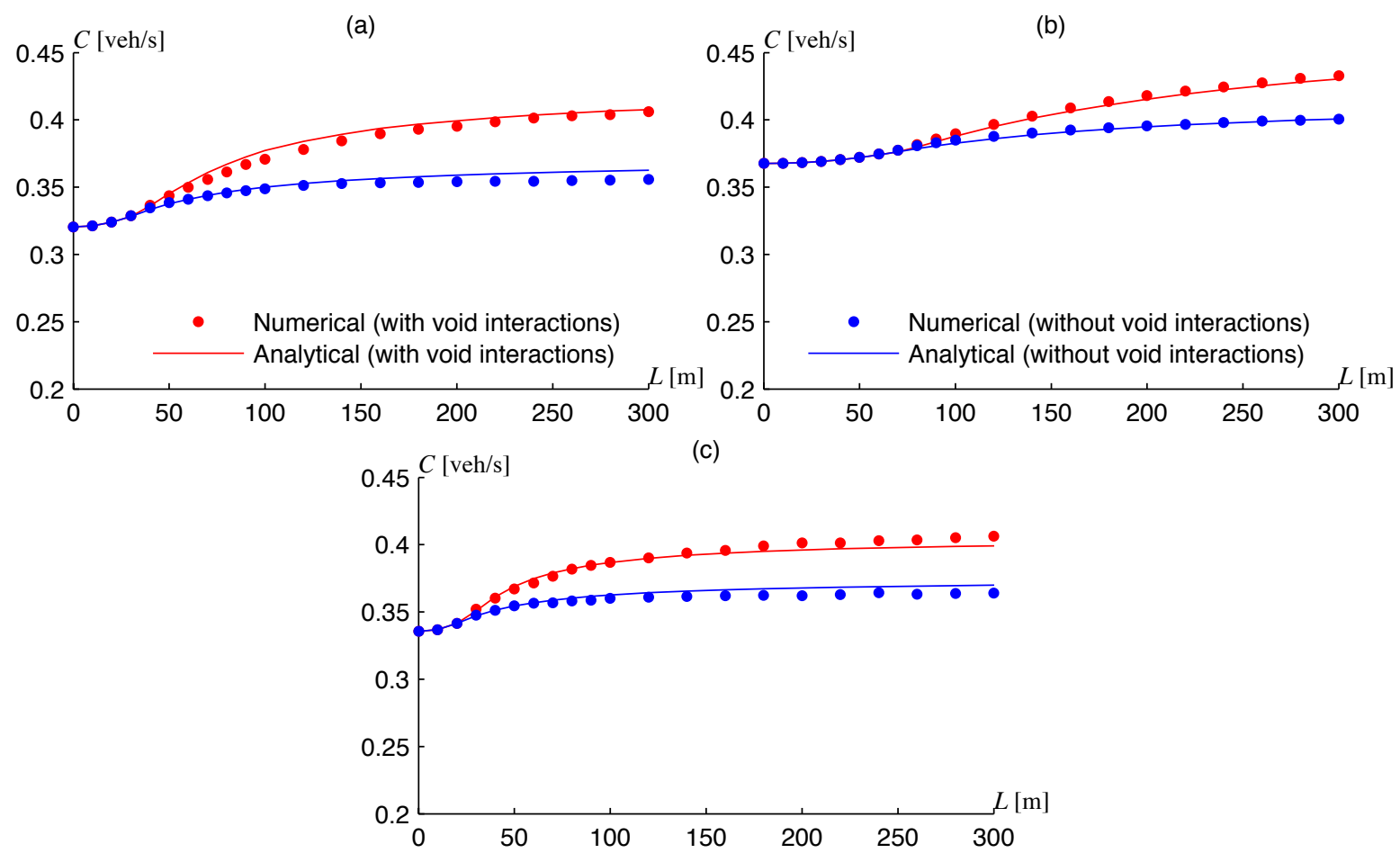

Figure 3: Comparison between analytical and numerical results when considering or not the interactions between waves and voids. (a) regular inserting flow: $q_{0}=0.174 \mathrm{veh} / \mathrm{s}$ (b) low inserting flow: $q_{0}=0.08 \mathrm{veh} / \mathrm{s}$ (c) high inserting flow: $q_{0}=0.26 \mathrm{veh} / \mathrm{s}$.

\section{Considering heterogeneous vehicle characteristics}

In this section, vehicle characteristics are no longer homogeneous, i.e. $s_{A}$ and $s_{K} \neq 0$. We still consider that the inserting speed is the same for all vehicles, i.e. $v_{0}$.

\subsection{Applying the generic expression}

To mimic realistic traffic conditions, we consider that inserting vehicles can be categorized into trucks and cars. The truck ratio is denoted $p^{*}$. Accelerations and jam densities are assumed to follow normal distributions for each vehicle class. Remember that the jam density formally describes in this framework the characteristics of the platoon upstream of an inserting vehicle. Because platoons are small, we distribute the jam density depending on the leader class, i.e. the inserting vehicle. Parameters related to trucks are identified using an asterisk $\left(^{*}\right)$ while those related to cars are identified using an apostrophe ('). For numerical examples, the following values will be applied: $p^{*}=0.2, \quad a^{*}=1 \mathrm{~m} / \mathrm{s}^{2}, \quad s_{A^{*}}=0.2 \mathrm{~m} / \mathrm{s}^{2}, \quad a^{\prime}=2 \mathrm{~m} / \mathrm{s}^{2}, \quad s_{A}=0.5 \mathrm{~m} / \mathrm{s}^{2}, \quad \kappa^{*}=67 \mathrm{veh} / \mathrm{km}, \quad s_{K^{*}}=10 \mathrm{veh} / \mathrm{km}$, $\kappa^{\circ}=145 \mathrm{veh} / \mathrm{km}, s_{K}=30 \mathrm{veh} / \mathrm{km}$.

Considering random accelerations and jam densities does not modify the global physical process described in section 2 and 3 when vehicles are inserting as far as the wave speed is constant, see Figure 1d. Thus, eq. (4) can be directly applied as soon as the different moments are properly determined.

\subsection{Determining the different moments}

$\boldsymbol{K}$ and $\boldsymbol{A}$-distributions. Those distributions are defined given the vehicle class. Applying the law of total expectation to the two first moments makes it possible to determine their means and standard deviations for the total population:

$$
\left\{\begin{array}{c}
a=p^{*} a^{*}+\left(1-p^{*}\right) a^{\prime} ; \kappa=p^{*} \kappa^{*}+\left(1-p^{*}\right) \kappa^{\prime} \\
s_{A}^{2}=E\left(A^{2}\right)-a^{2} ; \quad E\left(A^{2}\right)=p E\left(A^{* 2}\right)+\left(1-p^{*}\right) E\left(A^{\prime 2}\right) \\
E\left(A^{* 2}\right)=p^{*}\left(a^{* 2}+s_{A *}^{2}\right) ; \quad E\left(A^{\prime 2}\right)=\left(a^{\prime 2}+s_{A^{\prime}}^{2}\right)
\end{array}\right.
$$

Recall that there is no need to calculate $s_{K}$ because it has no influence on the effective capacity.

Probability for interacting $p_{\text {int }}$. The only modification when calculating $p_{\text {int }}$ is that the acceleration of 


\section{Leclercq et al}

the upstream closest neighbor is random. However, we can use at first glance the mean acceleration for this calculation. Eq. (7) and (8) remain unchanged, see Figure $4 \mathrm{~b}$.
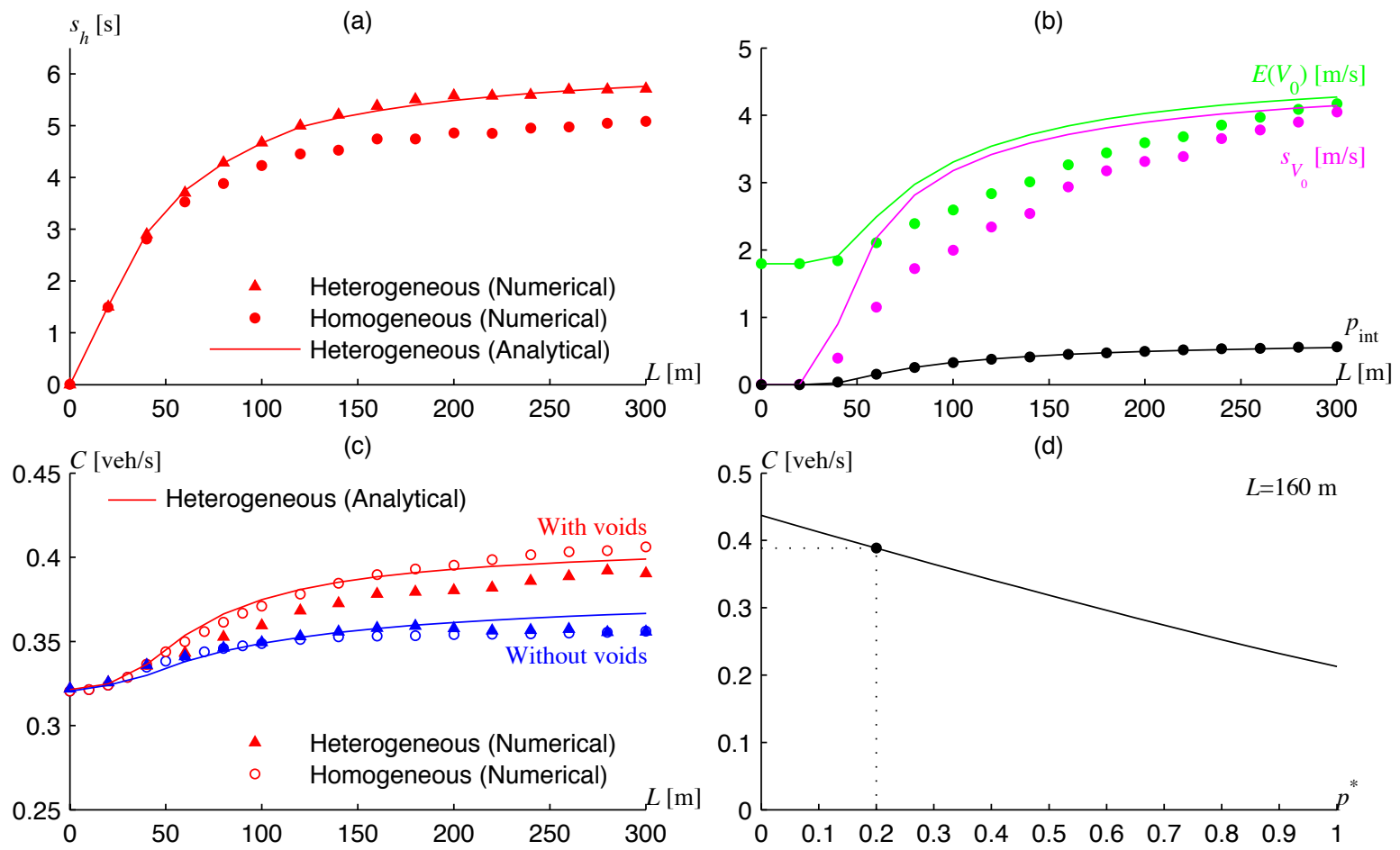

Figure 4: Integrating heterogeneous vehicle characteristics. (a) Standard deviation of $H$ (b) Mean and standard deviation of $V_{0}$, probability for interacting (c) Effective capacity $C$ with respect to $L$ (d) Effective capacity $C$ with respect to the truck ratio.

$\boldsymbol{H}$-distribution. A new phenomenon occurs when looking at the $H$-distribution with heterogeneous vehicle characteristics: some voids never disappear. It is clear in Figure 1d that if $a_{i}>a_{l}$ the upstream void boundary will join the downstream void boundary because initial speed at points A and B are the same. In the reverse situation, some cases may happen where the curve $\mathrm{BC}$ has no intersection point with the curve AC. In that case, if a wave coming from vehicle $l$ interacts with this void, it will never reach $x=0$ and should be disregarded. The effective occurrence of such a situation when $a_{i}<a_{l}$ depends on the relative position between $\mathrm{B}$ and $\mathrm{A}$ and thus depends on $v_{1, k}$ and the relative inserting positions of vehicle $i$ and $l$. Using geometrical considerations, it can be derived that the condition for the void created by vehicle $i$ not to disappear is:

$$
a_{l}^{2}+\left(a_{i}-a_{l}\right)\left(a_{k}+a_{l}+2 a_{k} \tau\left(h_{k}, v_{0}, a_{k}\right) / \tau\left(h_{i}, v_{1, k}, a_{i}\right)\right)<0
$$

By noticing that $v_{1, k}=a_{k} \tau\left(h_{k}, v_{0}, a_{k}\right)+v_{0}$ and using a first order Taylor expansion, it is possible to approximate the mean value for the ratio of $\tau$-functions in Eq.(14a) by $\sqrt{2}$. Eq. (14a) can then be further simplified:

$$
(2 \sqrt{2}-1)\left(a_{l}-a_{i}\right) a_{k}>a_{i} a_{l}
$$

Deriving analytically the probability for eq. (14b) to be true with respect to the $A$-distribution is too difficult. However, eq. (14b) provides an interesting insight. It appears that eq. (14b) will never be true if $a_{l}$ is not considerably higher than $a_{i}$. Thus and at first glance, we can consider that a condition for eq. (14b) to be true is that $i$ is a truck while $l$ is a car. Thus, the probability $p_{v}$ for a void not to disappear can roughly be estimated by:

$$
p_{v}=p^{*}\left(1-p^{*}\right)
$$

Finally, to determine the $H$-distribution, we assume that its behavior remains similar after disregarding the fraction of waves that are not reaching $x=0$. Such fraction is equal to the product of $p_{\text {int }}$ and $p_{v}$ (a wave should interact with a void that does not disappear). The new mean of $H$ is then equal to $E(H)=h_{0} /\left(1-p_{\text {in }} p_{\mathrm{v}}\right)$. We verify that $s_{H}$ can still be estimated by eq. (6) when $h_{0}$ is replaced by $E(H)$. Figure $4 \mathrm{a}$ shows the numerical results for $s_{H}$ with and without considering heterogeneous vehicle 


\section{Leclercq et al}

characteristics. Wave disappearances lead to increased $s_{H}$ values compared to the homogeneous case. It appears that the updated analytical eq. (6) provides an accurate fit for $s_{H}$ in the heterogeneous case. This result has been confirmed for multiple simulation runs with different parameters.

$\boldsymbol{V}_{\mathbf{0}^{0}}$-distribution. Random accelerations and the appearance of persistent voids strongly complicate the calculation of these moments. We need then to resort to strong assumptions. First, we consider that the distributions of $v_{1, l}$ can still be described by the distribution of $v_{1, k}$ in order eq. (9) still holds with $a=a_{k}$. Thus, the initial speed carried by the wave coming from vehicle $l$ only depends on the characteristics of vehicle $k$. Eq. (10) remains unchanged. For eq. (11), we have to consider in case of interactions that some waves never reach $x=0$. For $n$ inserting vehicles, only (1- $\left.p_{\text {in }} p_{v}\right) n$ waves crosses $x=0$. The fraction of waves carrying $v_{0}$ is then $r=\left(1-p_{\text {int }}\right) /\left(1-p_{\text {int }} p_{v}\right)$ while the fraction of waves carrying a random speed given by $V_{1}$ is $1-r=p_{\text {int }}\left(1-p_{v}\right) /\left(1-p_{\text {int }} p_{v}\right)$. Thus, eq. (11) should be updated into eq. (16).

$$
\left\{\begin{array}{l}
E\left(V_{0}\right)=v_{0}+\operatorname{ar} E(\tau(H)) \\
s_{V_{0}}^{2}=\operatorname{ar}\left(2 E(\tau(H))+a E\left(\tau^{2}(H)\right)\right)
\end{array}\right.
$$

Eq. (12) should also be updated because now two variables ( $H$ and $A$ ) are random in the $\tau$-function. We should then use the multivariate expression for the Delta method. We assume that $H$ and $A$ are not correlated. Thus, the new expression for the mathematical expectations of $\tau$ and $\tau^{2}$ are:

$$
\begin{gathered}
\left\{\begin{aligned}
& E(\tau)= \tau\left(h_{0}, v_{0}, a\right)+\frac{1}{2} s_{H}^{2} \frac{\partial^{2} \tau}{\partial H^{2}}+\frac{1}{2} s_{A}^{2} \frac{\partial^{2} \tau}{\partial A^{2}} \\
& E\left(\tau^{2}\right)= \tau^{2}\left(h_{0}, v_{0}, a\right)+\frac{1}{2} s_{H}^{2} \frac{\partial^{2} \tau^{2}}{\partial H^{2}}+\frac{1}{2} s_{A}^{2} \frac{\partial^{2} \tau^{2}}{\partial A^{2}} \\
& \frac{\partial^{2} \tau}{\partial H^{2}}, \frac{\partial^{2} \tau}{\partial A^{2}} \rightarrow(5) ; \frac{\partial^{2} \tau^{2}}{\partial H^{2}} \rightarrow(12) \\
& \frac{\partial^{2} \tau}{\partial A^{2}}=2\left(\frac{\tau\left(h_{0}, v_{0}, a\right)}{a}+\frac{w h_{0}}{a v\left(h_{0}, v_{0}, a\right)}\right)^{2} \\
&+2 \tau\left(h_{0}, v_{0}, a\right)\left(\frac{2 \tau\left(h_{0}, v_{0}, a\right)}{a^{2}}-\frac{2 w h_{0}}{a^{2} v\left(h_{0}, v_{0}, a\right)}\right. \\
&\left.-\frac{w^{2} h_{0}^{2}}{a v^{3}\left(h_{0}, v_{0}, a\right)}\right)
\end{aligned}\right.
\end{gathered}
$$

What is important when calculating the moments of $V_{0}$ from eq. (16) and eq. (17) is to use the initial $h_{0}$ value $\left(h_{0}=1 / q_{0}\right)$ and not the updated value $E(H)$. When calculating the $V_{1}$-distribution, we have to consider time intervals between waves coming from inserting vehicles whatever they are able to reach the boundary $x=0$ or not.

Figure $4 \mathrm{~b}$ shows the analytical calculations for $E\left(V_{0}\right)$ and $s_{V 0}$ compared to the simulation results. The analytical expression provides very accurate estimates for low and large $L$ values. In between, the discrepancies already observed for the homogeneous cases are amplified. They are also observed for a wider range of $L$ values. The reasons are twofold: (i) stronger assumptions have been made and (ii) in the heterogeneous case some voids can expand more and the probability that vehicle $l$ inserts within the void created by vehicle $i$ increases. The second reason is the main explanation for lower simulated values compared to the analytical predictions. However, a proof of consistency here is that the analytical expression provides close results when $L$ is large $(L>250 \mathrm{~m})$, i.e. when (ii) is very less frequent. Furthermore, we will see that the discrepancies observed for the $V_{0}$-distribution have a limited influence when calculating the effective capacity.

Covariance between $\boldsymbol{A}$ and $\boldsymbol{K} . \theta_{A, K}$ can be easily derived from the conditional behaviors of $A$ and $K$ with respect to the vehicle class. We simply further assume that $A$ and $K$ are not correlated within each specific class because trucks have specific acceleration capabilities while car accelerations are more related to the engine power than to the vehicle length. Otherwise, we would have to calibrate such correlations. 


$$
\theta_{A, K}=E(A \mathrm{~K})-a \kappa ; \quad E(A \mathrm{~K})=p^{*} a^{*} \kappa^{*}+\left(1-p^{*}\right) a^{\prime} \kappa^{\prime}
$$

Covariance between $\boldsymbol{H}$ and $\boldsymbol{V}_{\mathbf{0}}$. As for the homogeneous case, extensive numerical simulations show that $\theta_{H, V 0}$ is very low and can be neglected, i.e. $\theta_{H, V 0}=0$.

\subsection{Influence of vehicle heterogeneities on the effective capacity}

The final calculation for the effective capacity can be made using eq. (4) and the formulas eq. (13) to eq. (18). An important point is that $h_{0}=1 / q_{0}$ should be used in eq. (16) and eq. (17) when determining the moments of the $V_{0}$-distribution but everywhere else where $h_{0}$ is mentioned, one should read the mean value of $H$ and then used the updated expression $E(H)=h_{0} /\left(1-p_{\text {int }} p_{\mathrm{v}}\right)$. This is particularly true in eq. (4).

Figure $4 \mathrm{c}$ shows the results for the effective capacity and the reference scenario. Four numerical cases are presented whether void effect and heterogeneities are considered or not. Homogeneous cases use the proper means for the parameter distributions. Numerical results show that considering heterogeneity has no impact when voids are not considered. Introducing heterogeneous vehicle characteristics reduces the effective capacity when voids are considered but the magnitude is low. The maximum difference between heterogeneous and homogeneous cases appear for larger $L$ values and is never higher than $3 \%$. This has to be compared with the influence of voids that is in the order of $15 \%$. A very important insight here is that there is no need to complicate the calculation of the effective density by considering the distributions associated to heterogeneous behaviors. We only need to properly determine their mean values and applied the formulae for homogeneous case and voids.

Figure $4 \mathrm{c}$ also provides the results for the analytical calculation. They are not as close as for the homogeneous case but the discrepancies are always below $3 \%$ and can be considered fully acceptable. Furthermore, it appears that the difference with the analytical curve for the homogeneous case is hardly noticeable. This is because added terms in eq. (4) have finally very low values compared to those that already appear in the homogeneous case. Furthermore, those terms have opposite signs and nearly compensate. We can also note here that the discrepancies that appear when calculating the moments of $V_{0}$-distributions have very limited impacts even if they explain why the analytical calculations do not perfectly fit with the simulation when $L$ is between 50 and $250 \mathrm{~m}$. All these conclusions have been reinforced by large simulation tests.

Finally, Figure $4 \mathrm{~d}$ presents a direct application of the analytical approach: the effective capacity has been determined with respect to the truck ratio. Determining this curve is straightforward with the analytical formulae when it would have required extensive and painful simulation runs with classical modeling approaches.

\section{Conclusion and discussion}

This paper provides new analytical formulations for the effective capacity at active freeway merges. These formulations are able to account for interactions between voids that appear downstream of inserting vehicles and for heterogeneous vehicle characteristics. The main conclusion is that it is not necessary after all to introduce a detailed description of vehicle characteristics. A proper estimation of their mean characteristics is sufficient to derive an accurate analytical estimation of the effective capacity. This result is really appealing because it means that formulas presented in section 3 are sufficient. Such formulas are much more simple and can easily be implemented for practical applications. One important point is to consider the effect of voids interacting with waves. Recall that (Leclercq et al., 2011) provides all the methodological background to implement such updated analytical formulations into a full merge model that also account for situations where the on-ramp is not congested.

In this paper, three parameters have not been considered as random: the wave speed, the initial speed for inserting vehicles and the time interval between two insertions. Constant wave speed is required because it makes the physical process tractable, see Figure 1. Random time intervals between two insertions are straightforward to implement if we assume no modification in the calculations of $p_{\text {int }}, p_{v}$ and $V_{0}$ moments. In that case, only the standard deviation of $H$ has to be updated, see (Leclercq et al., 2011) for details. Otherwise, the problem becomes analytically intractable. Random speeds for inserting vehicles can be accounted in the framework of this paper but at the cost of much more complex analytical derivations. Here is some guidance about how to do it. First, the standard deviation 


\section{Leclercq et al}

of $V_{0}$ in eq. (4) should be updated to account for the distribution $V_{0}^{\prime}$ of the initial inserting speed. This distribution can be considered independent from the distribution of the initial speed at $x=0$ due to interactions between waves and voids. Thus, the variance of $V_{0}$ can be simply calculated as the sum of the variances of these two contributions. Second, every time we use $v_{0}$ for calculating the moments of $V_{0}$, it should be replaced by the mean value $v_{0}^{\prime}$ of $V_{0}^{\prime} . v_{0}^{2}$ should be replaced by $v_{0}^{\prime}{ }^{2}+S_{V^{\prime}}$. Third a new term should be added when applying the multivariate Delta method in eq. (12) and eq. (17). This term corresponds to the second derivative of $\tau$ and $\tau^{2}$ with respect to $V_{0}{ }_{0}$. The main challenge is to properly estimate $p_{v}$ because it influences the calculations of the moments of $H$ and $V_{0}$. As for random accelerations, analytical tractability is hard to maintain and strong assumptions would surely be necessary.
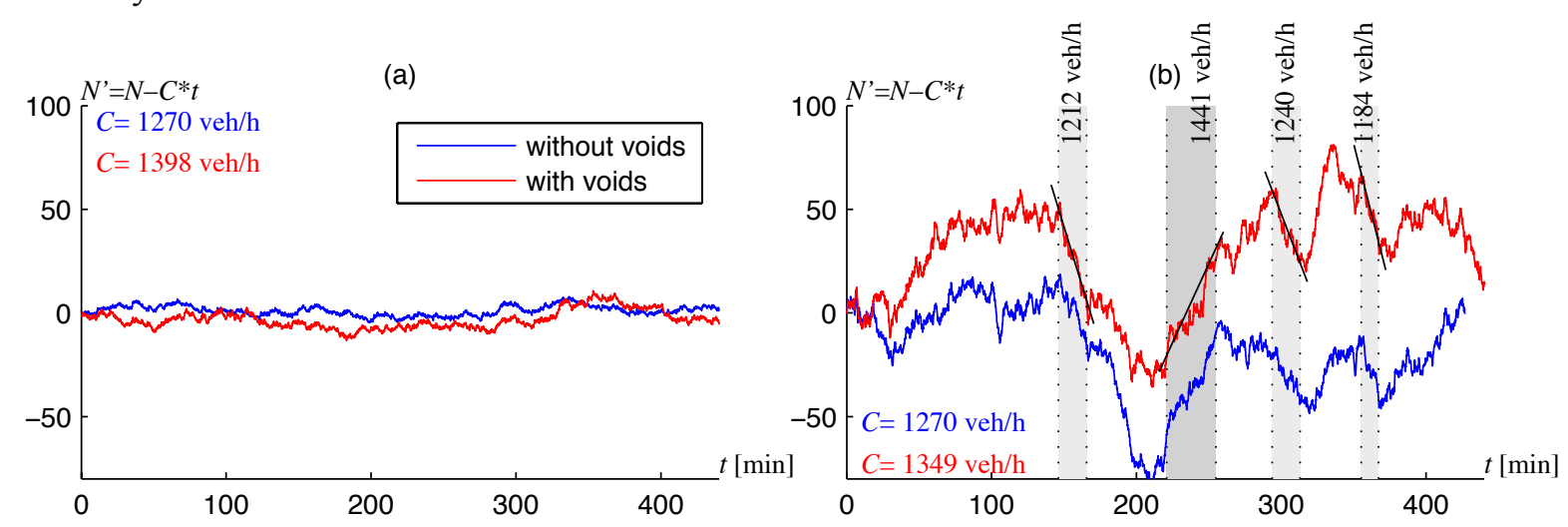

Figure 5: Numerical simulations for the flow dynamics at $x=0$. (a) Homogeneous vehicle characteristics (b) Heterogeneous vehicle characteristics.

We would like to provide a final comment about how important it is to consider heterogeneous vehicle characteristics. We have shown that this has no influence on the effective mean flow at an active merge. However, this may be crucial to represent other traffic phenomena. For example, Figure 5 provides the oblique cumulative count curves corresponding to our numerical simulations where voids effect and heterogeneities are considered or not. Figure 5a shows that when heterogeneities are not considered the flow dynamics at $x=0$ is very flat and close to the mean. When heterogeneous vehicle characteristics are implemented, the flow dynamics at $x=0$ is completely different even if the mean is nearly similar, see Figure 5b. Time periods with lower and higher flow values are alternately observed. The difference in the mean local flow between such periods is in the magnitude of 20 to $30 \%$ depending on the simulation runs for our reference scenario. This observation is very important because such flow variations can trigger stop-and-go wave appearance upstream of the merge. Figure $5 \mathrm{~b}$ shows that time periods with lowest flow values last between 10 and 20 min which is consistent with the duration of a stop-and-go waves. Accounting for heterogeneous vehicle behaviors appears then essential to catch such phenomena.

The authors currently investigate several further research directions. First, the proposed framework makes it possible to derive other indicators than the mean flow for the same physical process. This is notably the case of the variance of the capacity. Technically, the calculation of the variation requires the calculation of the mean value of $\delta_{\mathrm{i}}{ }^{2}$. A second-order Taylor expansion like in eq. (3) can be performed but the analytical expressions of the second derivatives of $\delta_{\mathrm{i}}^{2}$ with respect to the different variables are much more complex. We prefer not to overload the paper with lines of analytical expressions and resort to the simple simulations in Figure 5 to highlight the very important result related to heterogeneous vehicle characteristics. Note that the momentum of the different distributions that would be necessary for the derivations of others indicators are those provided in this paper. Second, the authors are working on a refined multilane extension to this framework compared to what is included in (Leclercq et al., 2011). This refined framework will include the effect of discretionary lane-changings that occur on freeway lanes. This will permit to determine not only the total effective capacity of multilane freeways but also to determine the related lane flow distributions in congestion. Note that such a distribution is considered homogeneous in (Leclercq et al., 2011) to derive the multilane capacity. This appears too restrictive compared to recent experimental findings (Reina and Ahn, 2014). Third, experimental validation of the analytical formulae is clearly the next goal. The 
experimental design is quite low because we only need flow measurements upstream of a merge including the on-ramp. The only point of attention is to verify that the merge is indeed a bottleneck, which requires a downstream detector with speed measurements. Because, real freeways have several lanes, we prefer to couple the experimental validation with the development of the multilane framework.

\section{Acknowledgements}

This research is sponsored by a visiting researcher grant from the Delft University of Technology Transport Institute, as well as the NWO project "There is plenty of room in the other lane". 


\section{References}

Carlson, R.C., Papamichail, I., Papageorgiou, M., 2014. Integrated feedback ramp metering and mainstream traffic flow control on motorways using variables speed limits. Transportation Research Part C, 46, 209-221.

Cassidy, M.J., Ahn, S. 2005. Driver turn-taking behavior in congested freeway merges, Transportation Research Record: Journal of the Transportation Research Board, 1934, 140-147.

Cassidy, M.J., Rudjanakanoknad., J., 2005. Increasing capacity of an isolated merge by metering its on-ramp, Transportation Research Part B, 39(10), 896-913.

Cassidy, M.J., Bertini, R.L., 1999. Some traffic features at freeway bottlenecks, Transportation Research Part B, 33(1), 1999, 25-42.

Chen, D., Ahn, S., Laval, J.A. Zheng., Z., 2014. On the periodicity of traffic oscillations and capacity drop: the role of driver characteristics, Transportation Research Part B, 59, 117-136.

Chiabaut, N., Leclercq, L., Buisson, C, 2010. From Heterogeneous drivers to macroscopic patterns in congestion, Transportation Research Part B, 44(2), 299-308.

Coifman, B., Kim. S., 2011. Extended Bottlenecks, the Fundamental Relationship and Capacity Drop. Transportation Research Part A. 45(9), 980-99.

Chung, K., Rudjanakanoknad, J., Cassidy, M.J., 2007. Relation between traffic density and capacity drop at three freeway bottlenecks, Transportation Research Part B, 41(1), 82-95.

Daamen, W., Loot, L., Hoogendoorn, S.P., 2010. Empirical analysis of merging behavior at a freeway on-ramp, Transportation Research Record: Journal of the Transportation Research Board, 2188, 108118.

Daganzo, C.F., 2005. A variational formulation of kinematic waves: basic theory and complex boundary conditions, Transportation Research Part B, 39(2), 187-196.

Daganzo, C.F., 1995. The cell transmission model, Part II: network traffic, Transportation Research Part B, 29(2), 79-93.

Kerner, B.S., 2002. Empirical macroscopic features of spatial-temporal traffic patterns at highway bottlenecks, Physica Review E, 65(4), 1-30.

Kim, S., Coifman, B., 2013. Driver relaxation impacts on bottleneck activation, capacity, and the fundamental relationship. Transportation Research part C, 36, 564-580.

Laval, J.A., Daganzo, C.F., 2006. Lane-changing in traffic streams, Transportation Research Part B, 40(3), 251-264.

Laval, J.A., Cassidy, M.J., Daganzo, C.F., 2005. Impacts of lane changes at on-ramp bottlenecks: a theory and strategies to maximize capacity. In: R. Kühne, T. Poeschl, T., A. Schadschneider, M. Schreckenberg, D. Wolf (Eds.), Traffic and Granular Flow '05'. Berlin: Springer, 577-586.

Laval, J.A., 2006. Stochastic processes of moving bottlenecks: Approximate formulas for highway capacity. Transportation Research Record: Journal of the Transportation Research Board, 1988, 8691.

Leclercq, L., Laval, J.A., Chiabaut, N., 2011. Capacity Drops at Merges: an endogenous model, Transportation Research Part B, 45(9), 1302-1313.

Newell, G.F., 1998. A moving bottleneck. Transportation Research Part B, 32(8), 531-537.

Leclercq, L., Chanut, S., Lesort, J.B., 2004. Moving bottlenecks in the LWR model: a unified theory, Transportation Research Record: Journal of the Transportation Research Board, 1883, 3-13.

Lighthill, M.J., Whitham, J.B., 1955. On kinematic waves II: A theory of traffic flow in long crowded roads, Proceedings of the Royal Society, A229, 317-345.

Oehlert, G.W., 1992. A note on the Delta method, The American Statistician, 46(1), 27-29.

Richards, P.I., 1956. Shockwaves on the highway. Operations Research, 4, 42-51.

Reina, P., Ahn, S., 2014. Prediction Of Merge Ratio Using Lane Flow Distribution. \#14-5459. Proceedings of the 93rd Transportation Research Board Annual Meeting (TRB). Washington: Transportation Research Board, 15p.

Sarvi, M., Kuwahara, M., Ceder, A., 2007. Observing freeway ramp merging phenomena in congested traffic, Journal of Advanced Transportation, 41(2), 145-170.

Srivastava, A., Geroliminis, N., 2013. Empirical observations of capacity drop in freeway merges with ramp control and integration in a first-order model, Transportation Research Part C, 161-177. 
Treiber, M., Kesting, A., Helbing, D., 2006. Understanding widely scattered traffic flows, the capacity drop, and platoons as effects of variance-driven time gaps, Physica Review E, 74(2), 1-10.

Zheng, Z., Ahn, S., Chen, D., Laval, J.A., 2011. Applications of wavelet transform for analysis of freeway traffic: bottlenecks, transient traffic, and traffic oscillations, Transportation Research Part B, 45(2), 372-384. 